\title{
Risk cyanobacterial toxins: Occurrence, ecology, detection, toxicology, and health effects assessment
}

This special issue of Toxicology and Applied Pharmacology not only is dedicated to the newest developments in understanding the presence and development of cyanobacterial toxins in the environment but also emphasizes the problems and recent advances in detection of these toxins. It quickly becomes quite apparent that a thorough understanding of the biology/ecology of the cyanobacteria, whether or not they produce toxins, the circumstances under which the production of toxins is promoted, and the type and concentrations of toxins that are produced in a single sample are of utmost importance for any type of exposure and health risk assessment. Any form of risk assessment requires an understanding of the toxicological profile inherent to these toxins, and it comes as no surprise that this is not a simple matter but rather complex due to the numerous different congeners of the cyclic peptide toxins possibly present in an environmental sample at any given time. Furthermore, the form and type of exposure, e.g., through water or food or even via the self-inflicted exposure to cyanobacterial products, allow largely differing risk scenarios. Although for some exposure regimens, e.g., drinking water and swimming in contaminated waters, risk and hazard assessment schemes have been developed, most of these are being hampered by the fact that the basic toxicological profile used for these schemes is that of one single cyanobacterial peptide congener, namely microcystin-LR. Critical evaluation of this situation reveals that this could be a major problem in the future, as the nearly 80 different microcystin congeners known to date have largely variable toxicokinetic characteristics and thus may also provide differing toxicodynamic profiles. Moreover, as multiple toxin congeners can occur in a single exposure situation, hazard and risk assessment would have to consider a mixture type of toxin exposure and not a single toxin exposure. Consequently, any useful hazard and risk assessment would have to take the latter factors into consideration, yet none of these have been critically considered in the present risk assessment formats. Furthermore, the only internationally available risk assessment for cyclic peptide toxins and ensuing guidance limit values are those from the WHO for microcystin-LR in drinking water.

In summary, this special issue of TAAP clearly delineates the breadth of knowledge on the ecology and biology of cyanobacteria, their potential, and their pathways for toxin production. However, it also highlights the dearth of information on the toxicology and the underlying mechanisms that can be used for hazard and health risk assessment and management. It is hoped that this TAAP special issue will be an incentive to invest more mechanistic research efforts into this topic so that an improved understanding can be gained in the near future.

Last but not least, I thank all of the contributing authors for their patience, motivation, and help in getting this special issue of TAAP together. I also express my gratitude to my family for their patience and endurance while I was editing and reviewing the numerous revisions of this special issue of TAAP. 